process of elongation has not stopped as soon as the pulps of these teeth have been removed; and, in a number of these cases, I have found in sealing of the ends of the roots, orifices that were larger than those often found in an ordinary tooth. 'I'hese are some of the instances that keep coming back to me in thinking of the practicable adaptability of these views, and the more I have thought of them the more have I been impressed that Mr. Constant's theory is the most scientific interpretation of the physiologic action of eruption of all heretofore presented. I feel that this section has been highly honored in being the vehicle of presenting this paper to the scientific world.

Mr. Constant-First, I thank you for your very kind remarks and the kind manner in which you have received the theory that I have placed before you. It would be impossible in the short time at my disposal to enter fully into every fact with regard to the eruption of the tooth. All that I desired to point out was that the very simplest fact in the whole problem had been ignored. It was not my intention to enumerate the things we did not know. When we say that we do not know why the ovum comes to the surface of the ovary, I think that there certainly must be something other than the mere mechanical force in connection with the eruption of the teeth. I think they are reasons we all fully recognize, but $I$ do not think they are reasons for putting forward the purely mechanical side and ignoring the other. Dr. Talbot stated that he did not think there was any difference in the removal of roots of temporary teeth by resorption and by absorption. On that point I disagree.

Dr. TalboT-I said in roots that had dead pulps and implanted teeth. |Dr. Talbot here reread the statement referred to. ]

Mr. Constant-I inferred from that that Dr. Talbot was of the opinion that whether a temporary tooth was alive or dead, exactly the same process of absorption occurred, but I see that it does not admit of that construction. My contention is that it is rather more than a question of degree. The appearances, so far as I have seen them, seem extremely different in the two instances. In the one case you get very sharply clear-cut nodules of the edge of the root. In the case of the root of a temporary tooth in which the pulp is being destroyed (I am speaking now entirely of the macroscopic appearance) the surface of the dead root is covered with thick membrane and the edges of the absorbed part are smooth. That is what I term the result of pathologic process of absorption. The physiologic process of resorption which takes place when the pulp is alive differs very markedly, that difference being the sharp margins and the absence of one thickened membrane, which has the greasy feel in the other case. Dr. Talbot speaks of the process of the removal of subadjacent tissue from the advancing crown as a process of inflammation. I do not like the term, inflammation, used in that connection. I do not want to enter into the point of exactly what takes place during the removal of subadjacent tissue, as I wish the simpler point to be cleared up first. Until we are in agreement as to the mechanical force which brings the tooth to the surface we can make no progress. Until we can determine the process by which the tooth comes up it is idle to discuss more complex questions. The point I wished to bring before you, and which $I$ have succeeded in making clear, is that mechanical part of the process which is the simplest and it is the part that has been ignored. That there is a mechanical force there can be no doubt in the mind of any dentist who has seen a tooth pressing on the gum before eruption. It has been urged that the tooth came up simply because the subadjacent tissues are removed; but that idea would be dismissed after close observation. In regard to a corroborative point, I ventured in the paper to touch on pathologic conditions, and I can assure you that since I have worked out this theory, some years ago, I have found no pathologic condition that has caused the slightest doubt in my mind as to the applicability of the theory. The point that the chairman mentioned and which would strongly confirm the view is, that in the case of a six-year molar, in which the pulp has been prematurely destroyed, one finds afterward that that tooth does not come up properly into line as do teeth in which the pulps have not been destroyed, and when it does it is not accompanied by any corresponding growth of the alveolaris; that is to say, there is slight extrusion, but not sufficient extrusion to bring it into occlusion with the opposing tooth.

DR. M. L. RHEIN-A point in corroboration of the fact that removal of the tissue is not sufficient to aid in the eruption of the teeth, is the occurrence that we have all seen so often, the premature lancing of deciduous teeth and the reformation of scar tissue, even though the lancing is done in the most thorough manner. I simply mention this as a corroborative point and one which is conclusively clear.

\section{SPLENIC ANEMIA;}

\section{REPORT AND DEMONSTRATION OF A CASE.*}

\section{J. LICHTY, M.D.}

Lecturer on Medicine and Physical Diagnosis in the Cleveland College of Physiclans and Surgeons; Visiting Physiclan

to the Cleveland General Hospital; Visiting Physician to the Cleveland City Hospital.

CLEVELAND, OHIO.

Mrs. S., aged 32, who was referred to me last fall through the kindness of Dr. Rosenwasser, gives the following history: The family history, including that of uncles and aunts and grandparents, is negative. She was born and reared in this city; has never lived in a malarial district; has had no venereal disease and was a healthy girl, never having had any serious illness. She was married at 26 , and has two healthy children. She has never had any miscarriages. In March, 1900, more than three years ago, she noticed that she was losing weight and strength, and that her feet and ankles were swollen. The abdomen also inereased in size, and both the feet and abdomen were tender to touch. The menses became irregular, appearing at intervals of six weeks, or three and sometimes even five months. These symptoms continued more or less uniformly for more than two years, though the abdomen seemed to increase in size constantly, so that she presented the appearance of a pregnant woman. She also soon noticed a large hard mass in her left side beneath the ribs. For two years also she was attacked with frequent attacks of severe bleeding from the nose. In July, 1902, one year ago, she was suddenly seized with a severe and serious gastric hemorrhage. She thought she vomited more than three pints of blood. Blood was also noticed in the stools for several days after. The hemorrhage, she said, caused more weakness and exhaustion than she had noticed at any time since the beginning of her sickness, and convalescence to her former condition was very tedious.

In September, 1902, she called on Dr. Rosenwasser, who referred her to me for an examination of her blood, as well as any other examination which I should care to make. The mass which she had noticed in her left side could easily be felt without complete removal of her clothes. It was in the splenic region, had the shape of the spleen, moved with respiration, was not very tender to touch and was supposed to be the spleen. The thought of a nice case of splenic leukemia at once presented itself. To my surprise, however, the blood was entirely different from that found in the usual case of leukemia. The hemoglobin was 50 per cent., the number of red blood corpuscles was 4,288,000, and of white corpuscles 4,000-a leucopenia instead of a leucocytosis. A differential count of white blood corpuscles showed nothing abnormal in the proportion of lymphocytes and polymorphonuclears found in health. Only a few eosinophiles were found, a few nucleated red corpuscles, no megaloblasts, and there was no poikilocytosis. The corpuscles were uniform in size.

The enlarged spleen, hemorrhages and the blood with a marked anemia, low color index and leucopenia, were the three important physical signs pointing toward the diagnosis of splenic anemia. Other physical signs noticed then and

* Read before the Cleveland Academy of Medicine. 
since are as follows: There is poor nutrition, pallor, no icterus and no cyanosis. The face shows considerable pigmentation, so that she does not appear anemic. The reflexes are normal and there is no glandular enlargement. The feet, ankles and legs as far as the knees are much swollen, though there is no pitting on pressure. The chest is small and the intercostal spaces are sunken. The abdomen measured 31 inches in circumference. The lungs were apparently normal. The physical signs of the heart were very interesting. The base of the heart is in the second intercostal space to the left and the apex in the fourth. The chest wall is so very thin that one can hardly say whether there is a palpable thrill or not over the heart. The heart is perhaps slightly enlarged, unless the thin chest wall only makes it appear so. In the second and third intercostal spaces to the left there is a loud systolic murmur not transmitted much beyond the area of the heart. It does not disappear nor seem to be modified much by change of position. At some of the subsequent examinations the murmur was much less distinct. I am unable to come to any satisfactory conclusion as to whether this is a hemic or organic murmur. (The same kind of a murmur has been found in several cases which I have collected, though in only one, that reported by Hawkins, was any lesion found at autopsy. In his case there was a stenosis of both mitral and aortic valves.) The liver extended from the fourth intercostal space to three-finger breadths below the costal border. It was smooth with a round edge and not painful. The spleen extended from the sixth intercostal space in the left axillary line and the eighth in the back, to the crest of the ilium and almost as far forward as the median line. A notch could easily be felt, if not seen, in the anterior border. The spleen also is smooth with round edges, not painful to touch, and is rather firm. The kidneys are not palpable. The lesser curvature of the stomach is about two finger breadths above the umbilicus and the greater curvature three or four below the umbilicus. The abdomen was very prominent and there seemed to be some free fluid in it. The urine was highly colored, acid, contained a trace of albumin, but no casts.

Dr. Rosenwasser prescribed Fowler's solution in increasing doses, and she seemed to improve at once. In November he referred the case to me for treatment. She was then feeling very much improved, though the physical signs were about the same as six weeks before, except that there was perhaps more anemia, hemoglobin 53 per cent., erythrocytes $3,252,000$, and leucocytes 3,500. The ascites was also more marked, the abdomen being 34 inches in circumference, and there was much more albumin in the urine with some casts. The swelling of the feet, however, was disappearing. It seemed that the albumin increased in quantity as the dose of the Fowler's solution was increased. When the ascites was most marked there was a scant, highly albuminous urine, and there was much diarrhea. In February the arsenic was discontinued. In March there was only a trace of albumin and no casts. Instead of arsenic she has been taking three times daily two drams of glycerinated extract of red-bone marrow. This has seemed to agree with her, and though the blood examinations are not so very much better, if any, she at the same time feels much better. For the diarrhea and ascites there were given three times daily five grains each of diuretin and salicy. late of bismuth. She was also given a very scant diet of bread, butter and milk only. Under this regimen she improved rapidly, the diarrhea and ascites having disappeared in less than a month when the abdomen was again 31 inches in circumference. In spite of her limited diet she has gained in strength. In April the menses appeared, the first time since November, 1902, lasting one week, nothing abnormal. She has menstruated again in May and June. She has not been confined to her bed since the hemorrhage of nearly a year ago. She calls at my office for treatment, does her own house work, has a good appetite, sleeps well, walks considerably, is not dyspneic, and is one of the most cheerful and hopeful patients to be found. Blood examinations during the last nine months are as follows:

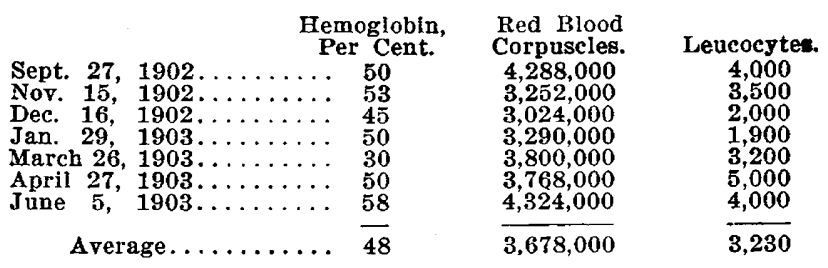

A differential count of leucocytes was also made the last time that she called at my office, a week ago. Altogether 250 leucocytes were counted, of which there were 12 large lymphocytes, 42 small lymphocytes, 182 polymorphonuclears and 14 eosinophiles. In this count there were also noticed 13 normoblasts and 10 megaloblasts. There was no poikilocytosis. The daily quantity of urine recently has been about 30 to 40 ounces, and the last specimen of urine examined was cloudy, acid, specific gravity of 1016, a small quantity of albumin, a few granular casts and considerable pus.

The term "splenic anemia" is not an old one. Many of the older, and even some of the newer, text-books do not use the term at all, though the terms "pseudoleukemia," "primary splenomegaly," "splenic anemia," and "Banti's disease" have been used more or less synonymously for some time. Wentworth says the name "splenic anemia" was "employed first by Gretsel and Greisinger in 1866 to describe cases of severe anemia associated with chronic enlargement of the spleen. Greisinger believed these cases to be the splenic form of pseudoleukemia." In $18 \% 0 \mathrm{H}$. C. Wood of Philadelphia reported several cases of pseudoleukemia, only one of which, however, was quite typical of the condition as considered in more recent years. Osler says that to Wood belongs the credit of first presenting the subject to the profession in this country. There were recognized two forms of pseudoleukemia previously, one in which the lymphatics alone were involved, and the other in which the lymphatics and spleen together were involved. Wood said: "I now desire to show that there is still a third form of pseudoleukemia-a splenic variety. Under the names, tumor of the spleen, splenic cachexia, etc., from time far back, medical records furnish accounts of cases which I believe represent this affection."

There is no doubt that one of Wood's cases was typical, though even that case had some glandular enlargement. And to him no doubt also belongs the credit of priority in this country which Osler gives him, though Woods himself did not use the term "splenic anemia," and his report appears as "A Case of Splenic and Lymphatic Hypertrophy Without Leucocythemia (Hodgkin's Disease, Adenia, Pseudoleukemia)," and it is probably on account of the title of his paper that the paper has been overlooked by some. In response to a note to Dr. Wood, in which mention was made of such credit belonging to him, he said: " $I$ was not aware that I had the honor you speak of in your letter, but I did know that my older papers had been absolutely overlooked by some among American literature on the subject." Since 1866 and $18 \% 0$, however, the term has been used quite frequently both here and in Europe, and a considerable number of cases have come into literature. The subject has been studied carefully by both physicians and surgeons. But with the single exception of Dr. Osler it has not been the fortune of any one to see more than a few cases. Both Wentworth and West have said within the last few years that aside from Osler's cases there are perhaps not more than 30 authentic cases reported. This number is no doubt too low. But in the last few years several new cases have been 


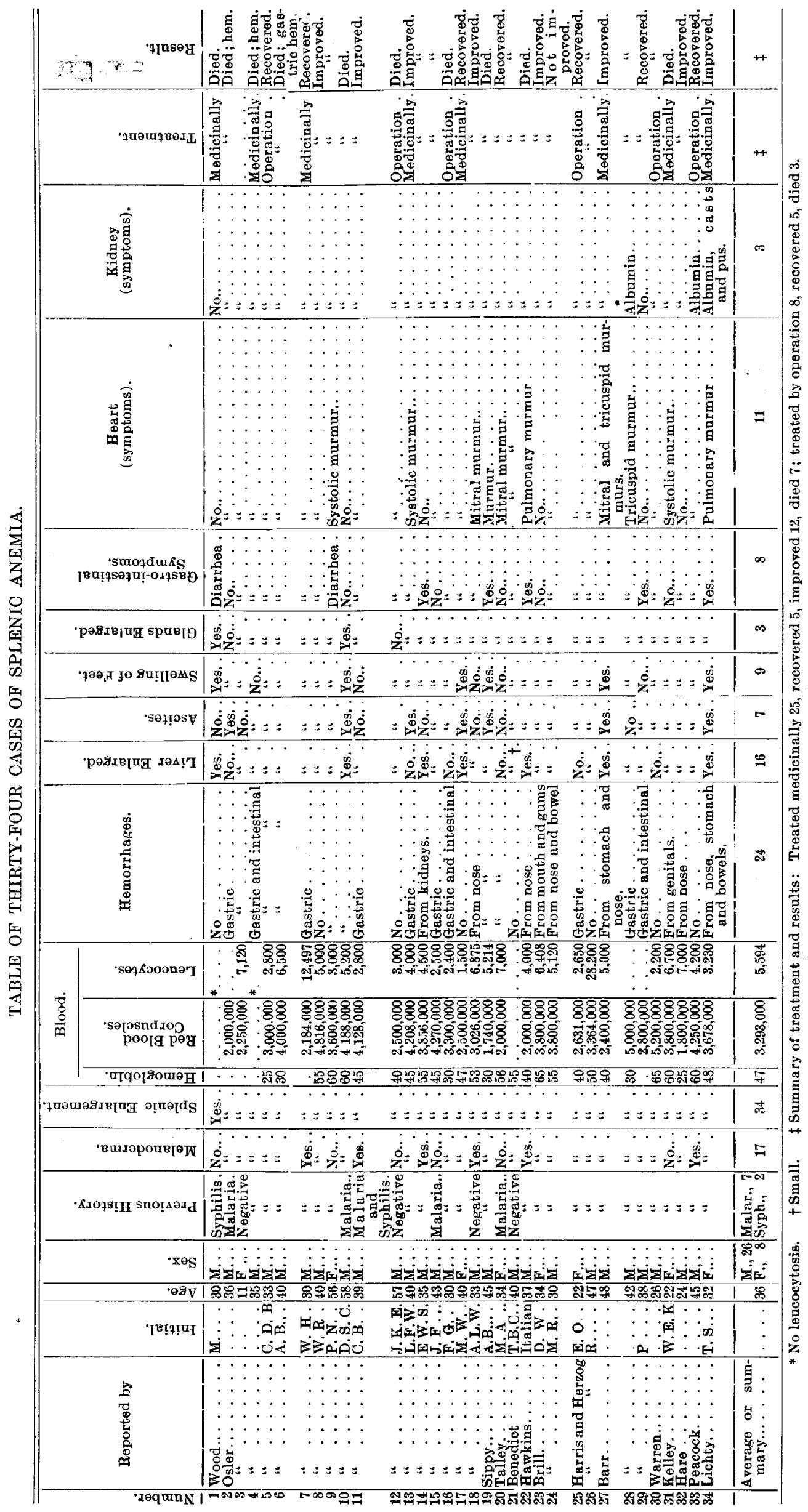


reported and I add one more to the list. The subject has been most thoroughly discussed at recent meetings of the Association of American Physicians. And though the name splenic anemia seems to have found a place in literature, some very good observers, including Musser and Stengle, still claim that the condition does not deserve a separate term and place. Wood in his original paper came to several conclusions, two of which I shall mention: 1, "Clinically, the so-called true and false leukemia are the same, save only in the matter of white blood corpuscles"; 2 , "all varieties of leukemia are represented in pseudoleukemia."

Osler has collected a series of 45 cases, excluding 17 of his own, from the members of the Association of American Physicians. Of these 45 cases 12 were of an acute type whose duration was from a few weeks to less than a year, 26 were of a chronic type, many of them having been afflicted for many years. The remaining 7 were not classified as either acute or chronic. Reports of these cases were submitted to him by such men as Dock, Musser, Cabot, Kelly, Herrick, Rotch, Wilson, Stockton and others of like recognition. From the limited report which he gives of these 45 cases (many of them perhaps unpublished) one is led to inquire whether all of them were in reality cases of undoubted splenic anemia. Some of them seem to be lacking the bulk of the physical signs of this condition.

In 1883 Banti described the condition and reported 3 cases. In 1894 he contributed further on the subject. In those papers and others published later especial reference is made to the enlarged spleen, also the enlarged liver and the absence of any glandular enlargement. On account of his evidently very accurate descriptions the malady occasionally has taken the name "Banti's Disease." While such credit may be due to Banti there are quite a few who believe that the term should be applied, or can be applied only to the latest and most marked stages of splenic anemia. We have, therefore, the terms "primary splenomegaly (found most frequently and typically in children and infants), "splenic anemia," and "Banti's Disease," all indicating perhaps different stages of the same condition, namely, a splenic variety of pseudoleukemia. To Osler belongs the credit, without any possible doubt, of having given to the profession the greatest number of most accurately reported cases of this kind. But the most complete description, perhaps, of the nature of the affection was given by Sippy of Chicago in 1899 ; and the most complete discussion of the literature on the subject, it seems to me, was given in 1901 by Wentworth of Boston. Wentworth, in a general way, discussed the subject "Association of Anemia with Chronic Enlargement of the Spleen," but many of his 88 references are to papers dealing almost exclusively with the subject of splenic anemia.

The symptoms of the disease are mainly anemia with low color index, and very commonly leucopenia; splenic enlargement often to the most marked degree; and a tendency to serious, and sometimes even fatal, hemorrhages. Other signs, as melanoderma, enlarged liver, ascites, swelling of the feet and legs, gastrointestinal symptoms, disturbances of the heart and kidney, irregular temperature and cachexia are also frequently present. The duration of the disease is from a few weeks to 15 years or more. You will notice that the case which I have presented showed about all these physical signs. Of the pathology nothing need be said here, and of the etiology only a word. Banti and others have regarded this as a primary anemia, and until we know of a more definite pathology and etiology of any of the primary anemias they can not be considered otherwise. With reference to spienic anemia, though, the profession seems to be looking toward the liver and portal circulation rather than toward the spleen for some causative factor. Barr thinks that the disease is secondary to vasomotor paresis of the abdominal circulation caused by some lesion in the visceral sympathetic ganglia. This theory has some support, though there are many who think that there are multiple causes rather than a single cause for all of this physical change.

The treatment of the disease has been both medical and surgical, of which neither seems to be very satisfactory. Harris and Herzog have collected reports of 12 recoveries from operations on 17 cases, the operation being, of course, splenectomy. To this number they added two more successfully operated cases. Osler has had four eases operated on with three deaths, hemorrhage having interfered much with the operations. Sippy is of the opinion that surgery alone is the proper treatment of this disease when the case is seen early. But he also says: "In reviewing the literature of splenectomy it is exceedingly difficult in many cases to determine the nature of the disease for which the operation was performed. As a rule, the operator has been more concerned in the technic of his operation than in the nature of the pathologic process for which it was performed." And though many patients pass through the danger of hemorrhage and shock from the operation, this does not prove that splenectomy removes the pathology of the disease in spite of the fact that many seem to improve after the operation. Somewhere I have seen the report of a case that died of gastric hemorrhage two years after a successful splenectomy and supposed cure of the case. Such a report adds strength to Barr's theory that the primary cause is not in the spleen, but in the visceral sympathetic ganglia.

Inasmuch as the case which I have reported has had much albumin, a number of casts and pus at times and as the disease is already of more than three years' duration, with a very much enlarged spleen and liver, with occasional ascites and much anemia, and inasmuch as the case has improved somewhat on a symptomatic treatment I hesitate to advise the risk of operation in her case, though appreciating fully the danger of another hemorrhage such as she had a year ago.

From considerable literature on the subject I have collected and tabulated the following cases, and will add the summary. Cases of primary splenomegaly in infants are not included in this list. All are undoubted cases of splenic anemia or Banti's disease, and with one exception all are adults. From what has been said before one would think it an easy matter to tabulate a hundred or more eases, and I must confess that this list is small, since half of the number has come from the writings of one man alone. But on account of the limited report which many of the authors give of their cases it has been found somewhat difficult to give a very large ccmplete report of cases in the time which has been at my command. However; the following data may be interesting to some. Of the 34 cases collected 26 were males, 8 females and the average age was 36 years. A history of malaria was found in but 7 cases and of syphilis in only 2 cases. Melanoderma was present in 17 cases. Splenic enlargement was well marked and the most prominent feature in all of them. Hemorrhage occurred in 24, hemorrhage from several sources 
in the same individual not uncommonly. Of these 34 cases 15 had hemorrhage from the stomach, 8 from the nose, $r$ from the bowels, 1 from the mouth and gums, 1 from the kidney and 1 from the genitalia. Next to splenic enlargement the anemia was the most striking symptom, being well marked in all of the cases, with a low color index and usually a leucopenia. In only two cases was there an excess of leucocytes. The average percentage of hemoglobin was 47 per cent., average number of red blood corpuscles $3,293,000$, and of white corpuscles 5,594. If we exclude the two cases in which there was a leucocytosis, the average number of leucocytes is 4,381. The liver was enlarged in 16 cases, small in one case (Benedict). Ascites was present in $\%$, swelling of the feet and legs in 9 , glandular enlargement in 3 , gastrointestinal symptoms in 8 , and cardiac murmurs, generally hemic, in 11 . In only two cases aside from my own is there any report of albumin in the urine and then "only a trace," while my case has had considerable albumin at times as well as some pus and casts.

The summary of the treatment and its results is most interesting. Of the 25 cases which were treated medically only 5 are recorded as "recovered," 12 were improved, 1 "not improved," and 7 died, hemorrhage having been the most common cause of death. Operaation was performed on 8 cases, of whom 5 recovered and 3 died.

\section{SQUINT.* \\ ALBERT B. HALE, M.D. chicago.}

Squint ought to be made an annually discussed subject at mothers' meetings, for it is through the mother that it must be detected, if this improper functional condition is to be corrected. It is not merely a question of two good eyes, although physiologically we need all our organs in the best of working order; cosmetically, the individual is nearer the type of perfect man if he not only sees with both eyes at once, but if the intended relationship of one visual axis to the other is constantly maintained; if no squint is observable. The young adult feels the effect of a cosmetic imperfection very keenly, and is grievously disappointed to find, when treatment or cure is sought, that the operation does not always lead to even cosmetic improvement, and that in the great majority of cases the squinting eye, if it does not persist in squinting, is, after all, a rather useless organ, on which no trust can be placed.

The correction of a squint, and if possible the restoration of function, must, then, be begun in early childhood. This is a fact not so much known, nor so usually accepted, even in our own profession-not even among all physicians who make a specialty of the eyeand the family counselor is sometimes guilty of saying that the child will outgrow its squint, or that the time to heal it, perhaps to operate on it, is about or after puberty. No greater error can be made. The time to treat a squint is as soon as it is noticed, which is very early in life, and the causes of the squint must be well understood before this advice is taken to heart and any treatment attempted.

Let me emphasize this statement: that many completely blind eyes do not squint-they jog along with their fellows content to obey the impulses from the brain without protest or discontent. On the other hand, most squinting eyes do see something, or did ear-

- Ragd before the Chicago Medical Society, 1908. lier in life, and at the beginning of the trouble they protested vigorously at the impulses from the brain and tried to act by themselves; tried to see something till they fell into bad habits, and long after they gave up trying to see accurately, they continued these bad habits and can not be reformed.

What, then, is squint? Of course, there is a paralytic squint, due to some acquired interruption to the nerve impulse or to injury to the muscle, but these are always characterized by double vision and usually come on later in life, through disease or trauma after the fusion faculty has been established. This may be called an organic lesion. The type to which I refer is, in contrast, called concomitant squint and has its origin in a functional disturbance, and the disturbed function is that of fusion. It arises early in life before the function is well established, and not being such a long-inherited function or instinct-for we know that binocular single vision is possessed by but few of the highest animalsit is easily disturbed. Generally, there is some exciting cause, such as a fever, a shock or fright, in childhood before the sixth year, while this habit is becoming acquired, and when weakened or lost, it is so delicate a function that neglect may often destroy it altogether, while to re-establish it demands attention, training, or the stimulus to spontaneous exercise of the habit, by placing the eyes in such relationship to each other that it is as easy to exercise as it is to neglect this function.

Herein lies the great error on the part of both physician and parent, the assumption that the child will "grow out" of the habit of squinting. Perhaps he will, but in the great majority of cases only at the expense of the vision of the squinting eye. Once have it well understood that treatment should begin early, as soon as the defect is seen, and there will be fewer squinters in the world, more sound eyes, and less complaint that the operation so seldom accomplishes what is expected of it.

The diagnosis is easy. Any layman can tell when a child or adult squints. but the etiology in the light of modern study shows that neither nerves nor muscles are primarily at fault. It is the brain, where the fusion function is located, and it is the brain we must first educate before we can hope ta control the eyes. Usually -not always-one eye will be found to see less distinctly than the other eye, the difference in degree varying with the individual and the duration of the squint. The result of disturbed fusion sense is imperfect innervation, exaggerated accommodative effort in one eye-squint.

The treatment resolves itself into three factors: (1) Correction of refractive errors; (2) training of fusion sense; (3) operation.

Of these, the first is the essential factor, and without it practically no case of squint can be overcome, or if the eyes do later in life grow straight, it will be at the expense of the vision of one eye.

'The second factor is of great importance, and implies such methods as the occlusive bandage, perhaps atropin to the good eye, stereoscopic, amblyoscopic and other visual exercises.

The third factor is operation, which is usually necessary, though squint may be entirely overcome without it. As a rule, however, some operation on a rectus muscle is necessary, and my later preference is for the advancement of a rectus muscle.

103 State Street. 